\title{
GHOST PARTICLES IN THE UNIVERSE NEUTRINOS IN ASTROPHYSICS AND COSMOLOGY
}

GEORG G. RAFFELT

Neutrinos are nearly massless and very difficult to detect because they interact so very weakly. Sixty years after seeing the first of these "ghost particles" we know a lot about their properties. Today, observing them in nuclear reactors, the Sun, the Earth's crust and atmosphere, and at high energies from distant cosmic sources is almost a routine task - they have become unique astrophysical messengers. They are important for a number of aspects: neutrinos shape some of the most dramatic astrophysical phenomena in the form of stellar-collapse supernova explosions, they may have created the excess of matter over antimatter in the universe, and neutrino-like «weakly interacting massive particles» may well account for the dark matter of the universe.

Keywords: neutrinos, dark matter, supernova, flavor oscillations, astroparticle physics.

Neutrinos, the «little neutral ones», are unique among elementary particles in that they neither feel the electromagnetic nor the strong force, the latter binding quarks to form protons and neutrons. So, neutrinos interact only by what is called the weak force and, of course, by gravity which is feebler yet. Wolfgang Pauli proposed neutrinos in 1930 as an explanation for the missing energy in nuclear beta decay and later famously commented that he had done a terrible thing by postulating a particle that could not be observed. Equally famously, he was wrong about this point, just as so many other assumptions about neutrinos had to be revised, notably their purported lack of mass. Actually, the 2015 Physics Nobel Prize was awarded precisely «for the discovery of neutrino oscillations, which shows that neutrinos have mass» and likewise the 2016 Breakthrough Prize in Fundamental Physics to five experiments for observing neutrino oscillations. This term refers to the circumstance that each of three neutrino types, or «flavors», transforms into another when propagating from the point of production (Bilenky, 2010; Gariazzo, Giunti, \& Laveder, 2016).
The electron $\left(v_{e}\right)$, mu $\left(v_{\mu}\right)$, and tau neutrino $\left(v_{\tau}\right)$ each is a quantum-mechanical superposition of three distinct mass values, causing the accumulation of phase differences along the neutrino path, and resulting in flavor conversion. The required distances for the conversion are macroscopic, from few to thousands of kilometers, revealing how tiny the neutrino mass differences are.

But Pauli was right that neutrinos are hard to measure. All astrophysical bodies emit neutrinos, but the brightest neutrino source in the sky is the Sun, producing two electron neutrinos for every helium nucleus fused from hydrogen, corresponding at Earth to 66 billion $\left(6.6 \times 10^{10}\right)$ neutrinos per $\mathrm{cm}^{2}$ and second. If we wanted to shield ourselves from this irradiation we would need several light years of lead. Yet the Sun is only eight light minutes away - no common material filling the space to the Sun could stop neutrinos. This of course also means that they are harmless to us.

The first solar neutrinos were observed in a pioneering experiment by Raymond Davis in the Homestake Gold Mine, South Dakota (USA), for which he received the 2002 Physics Nobel Prize. 


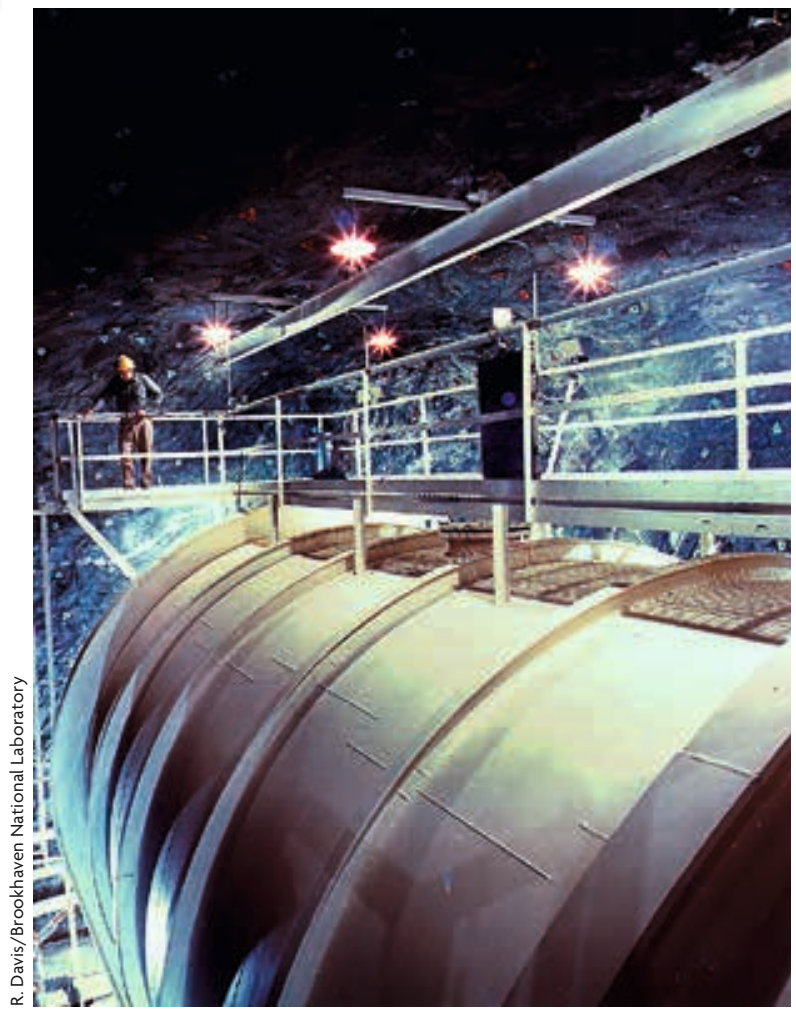

The first solar neutrino experiment in the Homestake Mine (South Dakota) built by Ray Davis, who received the 2002 Physics Nobel Prize for this achievement. The experiment took data in the period 1968-1994 and measured a total of some 800 solar neutrinos by capture on chlorine.

He used 600 tons of dry-cleaning fluid (tetrachloroethylene $\mathrm{C}_{2} \mathrm{Cl}_{4}$ ) which is cheap and contains a lot of the isotope ${ }^{37} \mathrm{Cl}$, which upon absorbing an electron neutrino turns to ${ }^{37} \mathrm{Ar}$. These single argon atoms, a noble gas, were flushed out and counted by their radioactive decays. Over the period 1968-1994, a total of some 800 solar were counted by this so-called radiochemical technique. The apparent deficit by a factor of 3 , in those days known as the «solar neutrino problem», was the first vestige of flavor conversion. Homestake could only see what survived of the original solar $v_{e}$ flux after partial conversion to the flavors $v_{\mu}$ and $v_{\tau}$. In 2002, the Sudbury Neutrino Observatory in Canada reported observing the full flux by a novel technique which responds to all flavors equally.

Homestake was an early case of «underground physics» in deep mines or tunnels under big mountains to shield rare-event experiments from cosmic rays. Today, an entire industry of neutrino and dark matter experiments fills many underground facilities on all continents. In Spain, the Canfranc Underground Laboratory (Huesca) in a former railway tunnel in the Pyrenees is a notable example. Historically, the earliest examples were the Kolar Gold Field (India) and the East Rand Proprietary Mine (South Africa), two miles underground, detecting a handful of neutrinos produced by highenergy cosmic rays in the upper atmosphere. The first of these «atmospheric neutrinos», the first-ever neutrino from a natural source, was measured on 23 February 1965, one decade after Frederick Reines and Clyde Cowan had detected neutrinos from a nuclear power plant in the US, an achievement belatedly honored with the 1995 Physics Nobel Prize.

\section{OBSERVING THE NEUTRINO SKY}

Today, the neutrino sky is watched by many large detectors, typically consisting of light sensors (photomultipliers) observing an active volume of water or mineral oil (Scholberg, 2012). Neutrino interactions produce charged particles that move faster than light in a material medium, which emit light either by the Cherenkov Effect (notably in water) or by the scintillation of certain organic compounds dissolved in mineral oil. The Baksan Underground Scintillation Telescope (200 tons scintillator), located in a cavity under Mount Andyrchy in the North Caucasus, began on 30 June 1980 and still operates today, followed in 1984 by the Liquid Scintillator Detector (90 tons) in a cavity of the Mont Blanc Tunnel, operating until the devastating tunnel fire of 1999. The first large water Cherenkov detectors (several thousand tons) were built in the early 1980s deep underground in the US and Japan to search for proton decay, a putative process that remains elusive even today. On 23 February 1987 they and Baksan observed instead about twenty events within a few seconds from the neutrino burst of supernova (SN) 1987A in the Large Magellanic Cloud at a distance of 160,000 light years. This historical event remains the only neutrino signal observed from a stellar collapse (Koshiba, 1992).

The Japanese detector Kamiokande in the Kamioka Mine (Kamioka Nucleon Decay Experiment) was followed by the cathedral-sized Super-Kamiokande $(50,000$ tons of ultrapure 


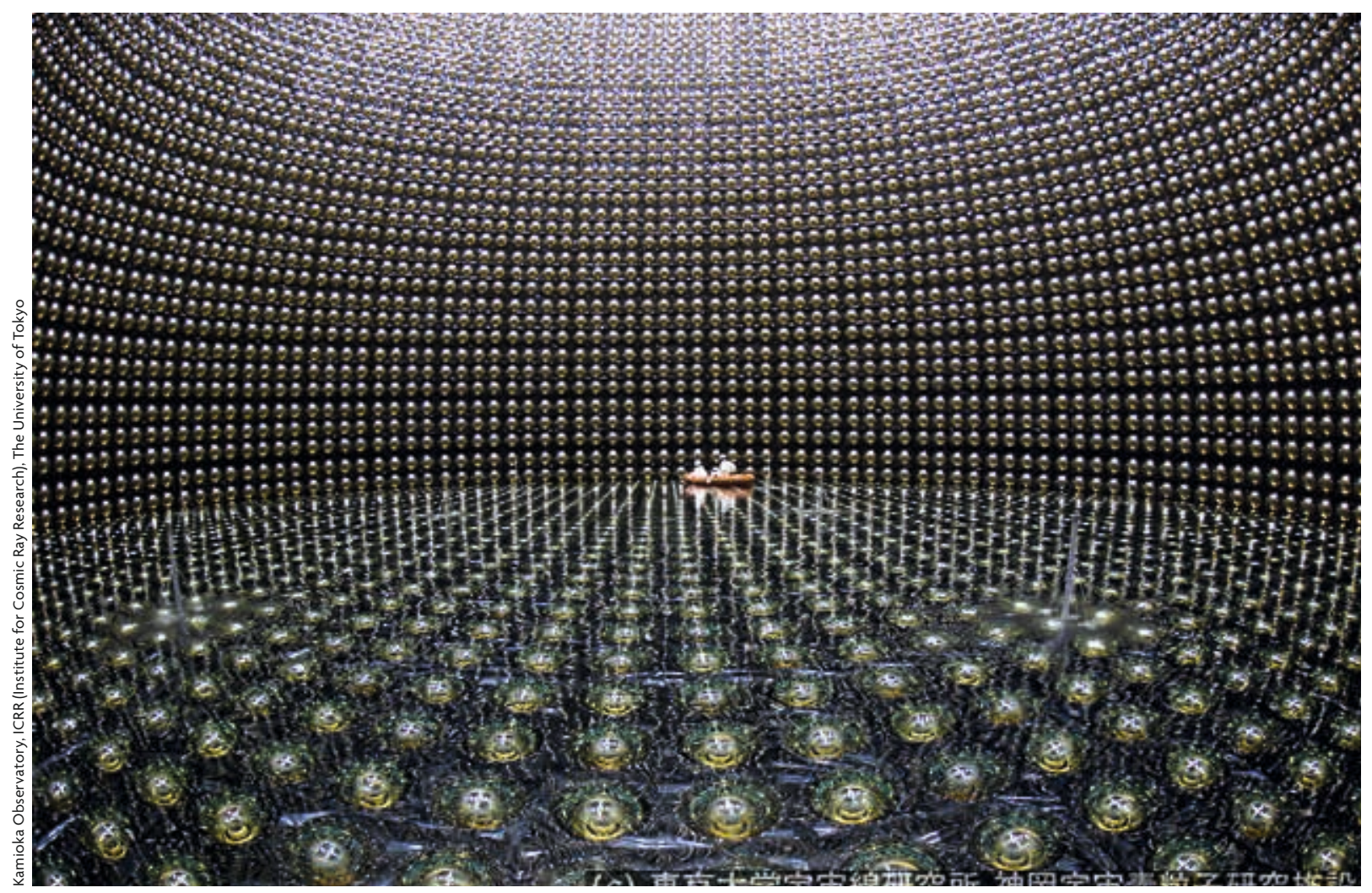

Super-Kamiokande neutrino observatory in the Kamioka Mine in Japan. The full underground tank contains 50,000 tons of ultrapure water, surrounded by 1,200 photo tubes. Shown is the floor at the beginning of filling after repairs (23 April 2006), ten years after it first began on 1 April 1996. This detector unambiguously identified neutrino flavor conversion of atmospheric neutrinos in 1998.

water watched by 1,200 photo tubes), taking up operations on 1 April 1996 and still running today after several repairs and upgrades. It has collected some 80,000 solar neutrinos, was the first to report flavor conversion of atmospheric neutrinos in 1998 , and serves to study neutrino flavor oscillations in a longbaseline experiment where a neutrino beam is sent from Tokai over $295 \mathrm{~km}$ across Japan to Kamioka, the Tokai-toKamioka or T2K experiment. It is the beauty of such large detectors that they function as observatories, serving multiple purposes at once. The next core-collapse SN in our galaxy, if it occurs at a typical distance of $10 \mathrm{kpc}$, will produce some 8,000 neutrino events in Super-Kamiokande and thus a very detailed glance at the physical processes of stellar core collapse.

At present, a yet larger water detector HyperKamiokande on the scale of a million tons is being developed (Hyper-Kamiokande Proto-Collaboration, 2015). The physics driver is to find out if neutrinos and antineutrinos oscillate the same. One expects subtle differences, a circumstance called $« \mathrm{CP}$ violation» (CP refers to the symmetry of physical laws under charge inversion plus inverse specular reflection), meaning that matter and antimatter (or neutrinos and antineutrinos) do not behave in perfectly symmetric ways.

The motivation to detect $\mathrm{CP}$ violation also drives the DUNE project, a new long-baseline oscillation program, where a neutrino beam will be sent from Fermilab near Chicago over $1,300 \mathrm{~km}$ to Homestake, refurbished as the Sanford Underground Research Facility (DUNE Collaboration, 2015). In a novel approach, the detector will use 20,000 tons of liquid argon.

The biggest-ever scintillator detector (20,000 tons), the Jiangmen Underground Neutrino Observatory 

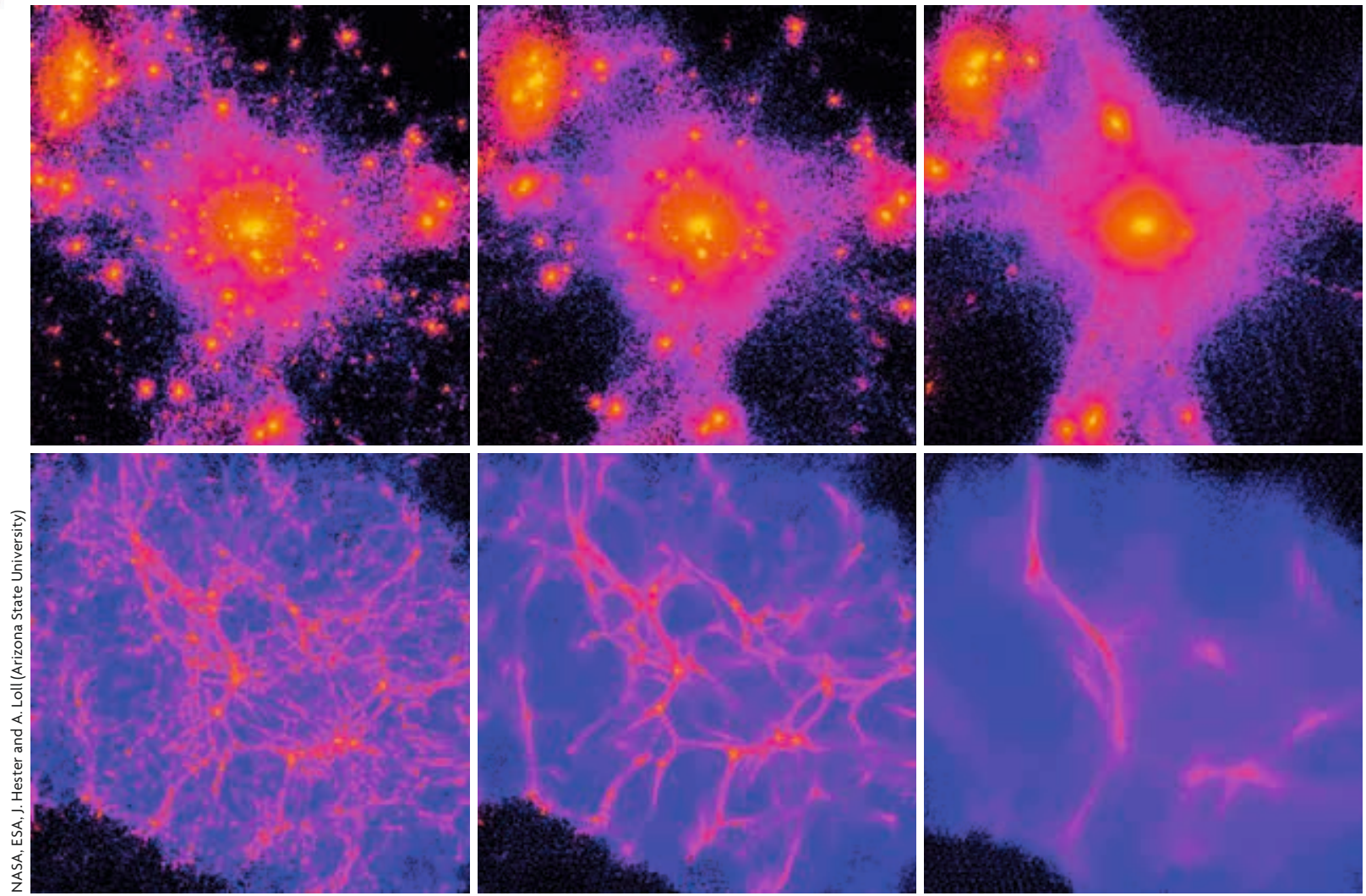

Simulation of the cosmic dark-matter distribution for cold, warm and hot dark matter (left to right; the first row zooms into one of the galaxy clusters that are observed in the second row). Neutrinos with small masses form hot dark matter and would wash out the small scale structure that is observed in the universe and corresponds to cold dark matter.

(JUNO) is being built in Southern China to measure reactor neutrinos at a distance of $53 \mathrm{~km}$ to solve another open question, the exact ordering of the three neutrino mass values (JUNO Collaboration, 2016). JUNO will be an excellent SN observatory as well. The largest existing scintillator detector is KamLAND (Kamioka Liquid Scintillator Antineutrino Detector) in Japan with 1,000 tons, which in 2002 used reactor neutrinos over a distance of 180 $\mathrm{km}$ to identify flavor oscillations and was the first to observe «geoneutrinos» from natural radioactive decays in the crust of the Earth, marking the beginning of neutrino geophysics.

Geoneutrinos were also observed by Borexino (300 tons scintillator) in the Gran Sasso National Laboratory near Rome. However, Borexino's main achievement was to measure the energy distribution of the solar neutrino spectrum with unprecedented precision and at lower energies than was previously possible, thanks to its low threshold and never before seen purity. In this way one is beginning to probe astrophysical details of the Sun not accessible otherwise.

The largest neutrino detector yet is IceCube at the South Pole. Phototubes in pressure vessels are attached to cables and sunk into 2,400-meter-deep holes drilled with hot water into the ice. After refreezing, the ice provides structural support to the matrix of photo sensors and also is the material to produce Cherenkov radiation. The detector was completed in December 2010, instrumenting a volume of one $\mathrm{km}^{3}$ with some 5,000 optical modules. The purpose is to find neutrinos at the highest energies produced by the cosmic accelerators that must exist in the universe to produce the high-energy cosmic rays which constantly clobber the Earth. Clarity about their sources will come from a yet bigger detector, the proposed IceCube-Gen2, with the tenfold volume. In 


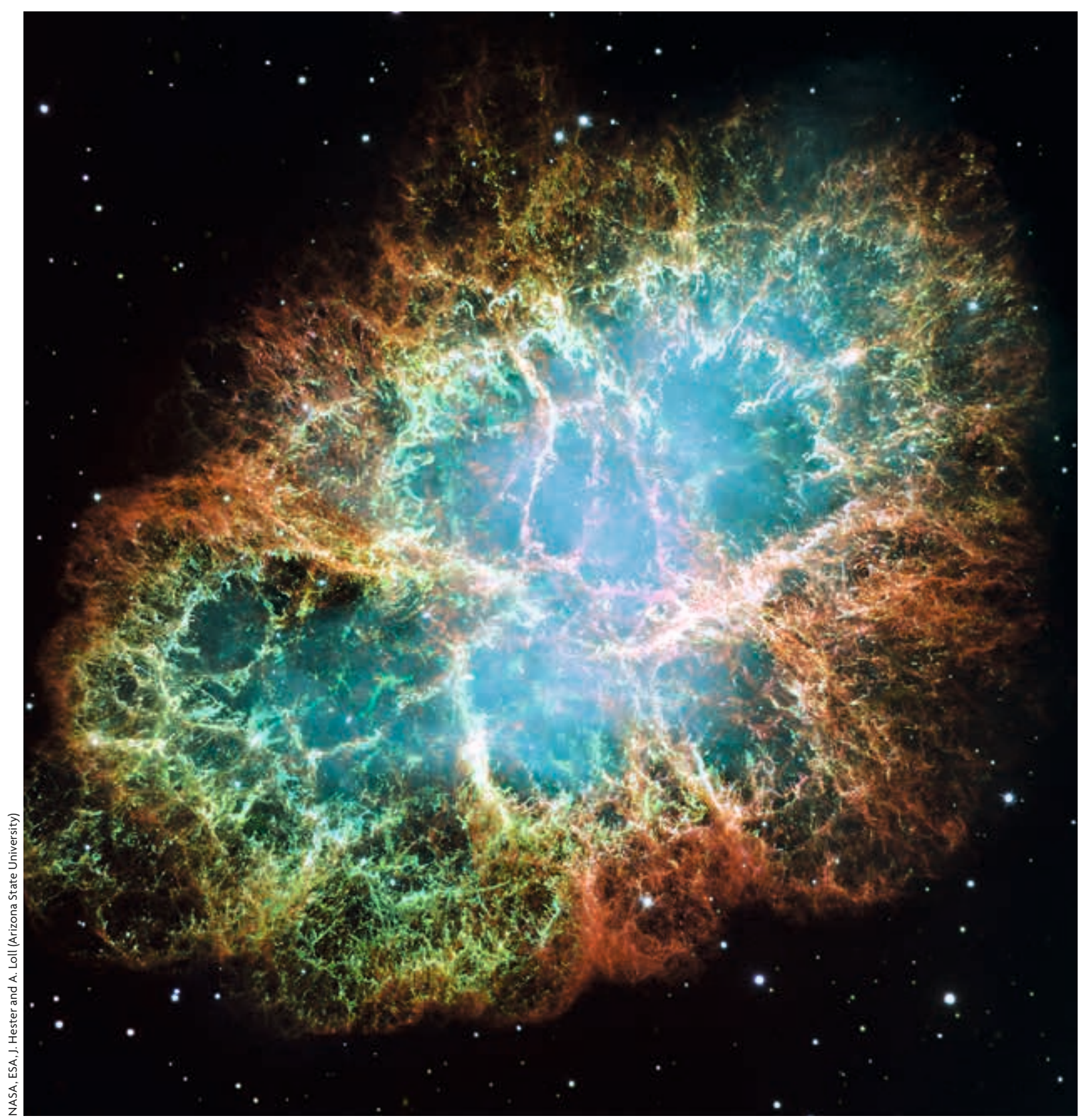

The Crab nebula is the remnant of the historical supernova of the year 1054 with the crab pulsar near its center being the compact remnant of the stellar collapse.

«THE NEUTRINO SIGNALS FROM

A THOUSAND GALACTIC SUPERNOVAE ARE ON THEIR WAY, SO EVENTUAL SUCCESS IS GUARANTEED. IT WILL BE A BONANZA OF SCIENTIFIC INFORMATION" parallel, a $\mathrm{km}^{3}$ detector may be built in Lake Baikal and a multi- $\mathrm{km}^{3}$ detector, or several large ones, in the Mediterranean, the KM3NeT project

These large-volume detectors are too coarse to identify Cherenkov light from low-energy neutrinos such as those from the Sun or Earth. However, IceCube is a fantastic SN observatory because the neutrino burst would make the ice glow in Cherenkov light and its sudden-burst profile would clearly set it apart from the steady background noise. In sea water, this background is too large caused by the natural abundance of radioactive potassium. 


\section{MONOGRAPH}

Violent universe

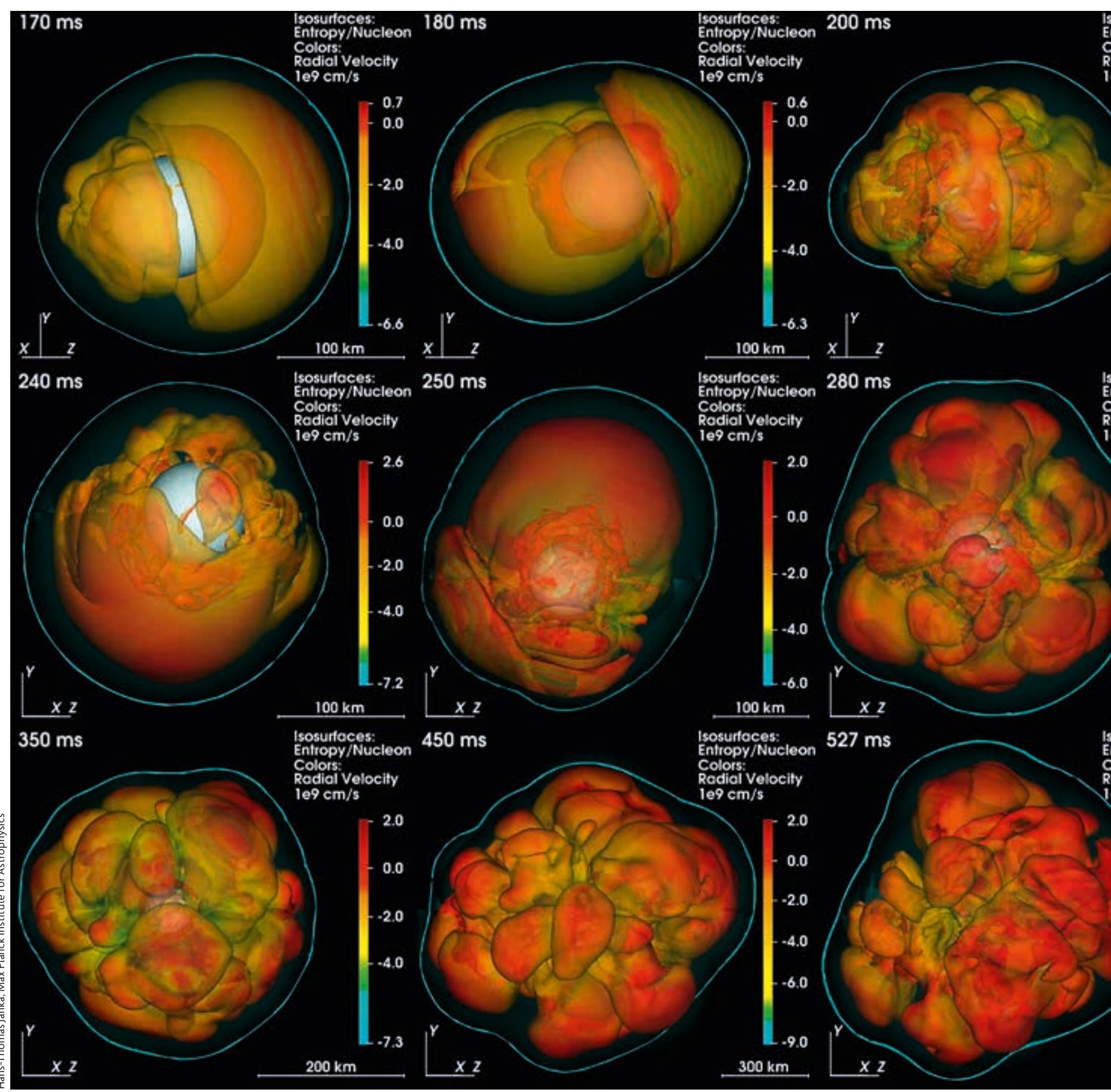

Supernova simulation in three dimensions of a twenty-solar-mass progenitor, showing sloshing and large-scale convection and eventually leading to a successful explosion. We can see different stages in the evolution of the material surrounding the protoneutron-star formed after the collapse. This material suffers convection (similar to what happens to boiling water) which results in all those bubbles in the time sequence. The outermost region in each image (marked with a green line) indicates the position of the shock wave, less and less close, as corresponds to an explosion and is indicated in the space scale of each image. 
In the next section, we discuss the astrophysical scenarios that could produce neutrinos in numbers and energies that are detectable by the devices described in prior paragraphs.

\section{COLLAPSING STARS AND SUPERNOVA EXPLOSIONS}

Massive stars at the end of their lives, after consuming all available nuclear fuel in their cores, must eventually collapse, typically forming a neutron star which often shows up as a pulsar. The stellar core of 1-2 solar masses eventually shrinks to a radius of 12-15 km, liberating gravitational binding energy corresponding to some $15 \%$ of its mass. Most of this huge amount of energy escapes over a few seconds in the form of neutrinos and antineutrinos of all flavors. The collapsed stellar core, having the density of an atomic nucleus, is so dense that even neutrinos are trapped and can only escape by diffusion, with a mean free path of a few meters.' Yet they are the ones which get out most easily and thus carry away most of the energy.

Neutrinos are instrumental in exploding the $\mathrm{SN}$ following the collapse. When the core has collapsed to nuclear density it becomes suddenly very stiff, a shock wave forms at its edge, and moves outward to eject the outer material. Yet the bounce shock halts, running against the ram pressure of the in-falling material, and perhaps in a quarter of all cases re-collapses to form a stellar black hole, an event called a «failed SN». In most cases, however, the shock wave gets revived by energy deposition of neutrinos which stream away from the core. This «delayed explosion mechanism» or «neutrino mechanism» or «Bethe-Wilson-mechanism» is the standard paradigm for core-collapse SN explosions (Janka, 2012), although other ideas exist. The neutrino observations of SN 1987A have confirmed the idea of neutrinos being trapped and escaping over some seconds, but a real smoking-gun confirmation of these ideas could only come from a high-statistics neutrino observation which the community is eagerly awaiting.

While existing detectors are large, we would need a $\mathrm{SN}$ in our own galaxy or one of its small satellites

\footnotetext{
1 The mean free path of a particle is described as the distance it can travel without interacting. The lower it is, the more interactions are produced.
}

such as the Magellanic Clouds, with an overall rate of a few per century. The operation of many large detectors over several decades for other purposes highlights the synergy between, say, long-baseline flavor oscillation studies and watching for the next galactic SN. The wait may be long, but it could happen any day. The neutrino signals from a thousand galactic SNe are on their way, so eventual success is guaranteed. It will be a bonanza of scientific information (Mirizzi et al., 2016). Several neutrino observatories are linked by the Supernova Early Warning System (SNEWS) and will issue an early warning to the astronomical community. ${ }^{2}$

Supernovae are relatively rare in individual galaxies, yet the long-term average energy released in neutrinos is about the same as that released in light by all stars. Stellar neutrinos and photons each amount to some $10 \%$ of the overall cosmic radiation density, the bulk being the cosmic microwave background. The relic neutrinos from the big bang, some 336 per $\mathrm{cm}^{3}$ today, represent a small part of the cosmic dark matter. Therefore, the Diffuse Supernova Neutrino Background (DSNB) from all past $\mathrm{SNe}$ is the largest cosmic neutrino radiation density. It is extremely hard to detect, but an upcoming upgrade of SuperKamiokande and the nascent JUNO detector may achieve this feat, extending neutrino astronomy to the edge of the visible universe.

\section{DARK MATTER}

The gravitational dynamics of galaxies, larger structures, and the overall cosmic expansion reveal large amounts of dark matter which cannot be ascribed to normal stuff known on Earth. Neutrinos have masses, but very small ones, meaning that after production in the hot early universe they would have slowed down very late and thus, by their free streaming, would have levelled density fluctuations which are required to initiate galaxy formation. Therefore, neutrinos would form what is called «hot dark matter», whereas galaxy formation requires the «cold» variety which moves slowly early on. Modern cosmological precision observations actually imply a restrictive limit on the allowed fraction of hot dark

\footnotetext{
2 Under http://snews.bnl.gov/ anyone can sign up to this service.
} 


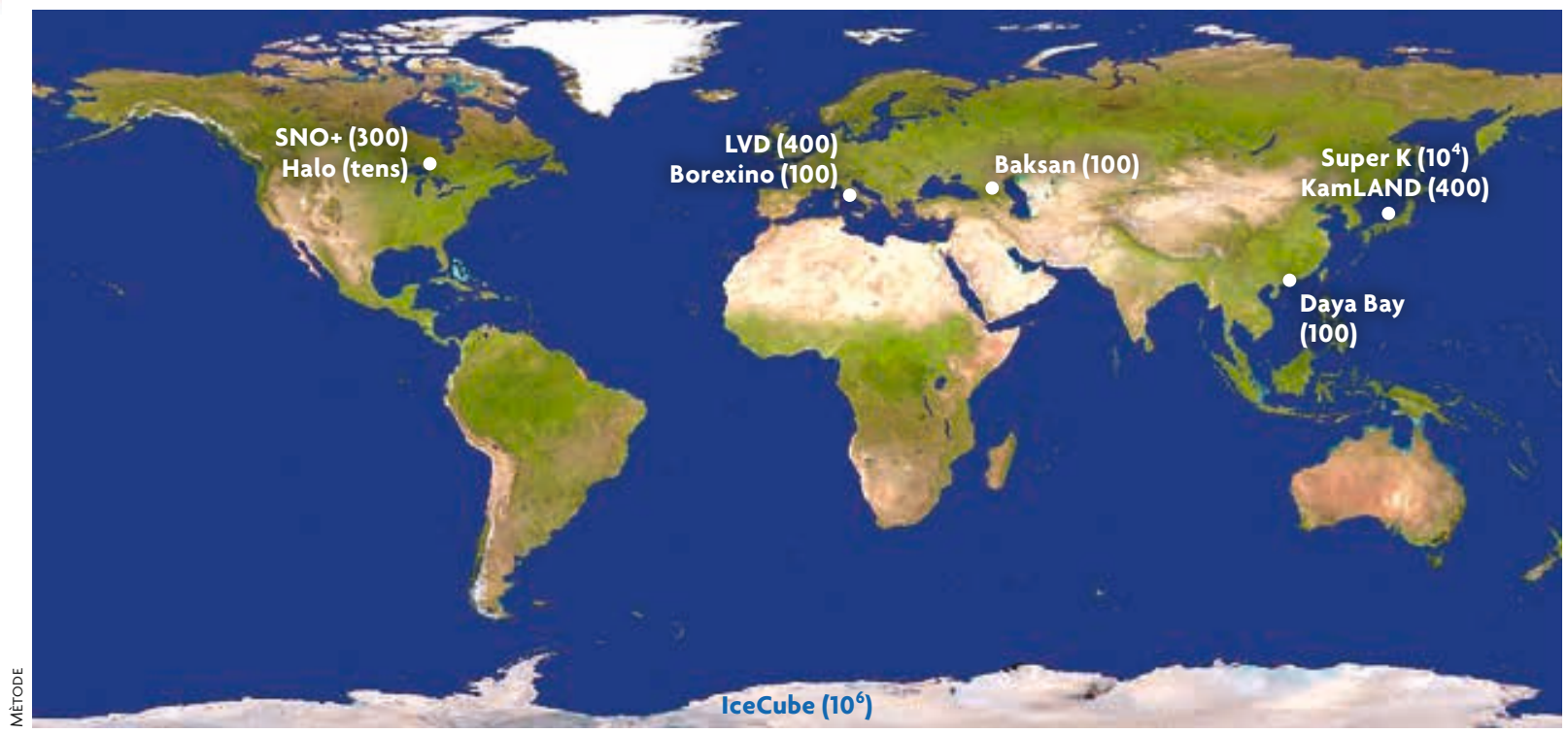

World map of existing large-scale neutrino observatories. In brackets the number of neutrino events expected from the next galactic supernova if it is at a typical distance of $10 \mathrm{kpc}$. Several even larger detectors are under discussion or under construction.

matter and thus on the possible range of neutrino masses. Intriguingly these upper limits are close to the neutrino mass differences implied by flavor oscillations (Villaescusa-Navarro, Bull, \& Viel, 2015). The next round of improved cosmological surveys, notably the forthcoming Euclid mission, may reveal the absolute neutrino masses in the sky. Of course, they could also find a contradiction with flavor oscillation experiments, posing new fundamental questions.

Meanwhile the physical nature of dark matter remains elusive. One long-standing hypothesis holds that it consists of novel weakly interacting massive particles (WIMPs) which in many ways resemble neutrinos, except for having much larger masses and yet weaker interactions (Bertone, 2010). Such particles are motivated by various ideas in theoretical particle physics and could be found, for example, at the Large Hadron Collider (LHC) at CERN, the world's largest particle accelerator. They could also show up indirectly. For example, they could accumulate in the Sun, produce high-energy neutrinos by annihilation, which could be detected by the IceCube neutrino telescope. Many direct search experiments look for tiny energy depositions by galactic dark matter WIMPs which would occasionally recoil from nuclei in various target materials. Such rare-event searches are prime users of underground laboratories worldwide.

No WIMPs have been found yet, raising some doubts about this paradigm. However, the search is not over, although at some point the detectors will become so large and so sensitive that they begin to measure solar neutrinos and backgrounds caused by atmospheric neutrinos. This circumstance is called the «neutrino floor» for dark matter searches. A traditional dictum has it that «yesterday's sensation is today's calibration, and tomorrow's background». It still seems preposterous that solar neutrinos might become an obstacle to scientific progress.

Supernova neutrinos have energies only slightly larger than solar neutrinos. So the neutrino

burst from the next nearby $\mathrm{SN}$ can be seen in the next round of large WIMP dark matter detectors, revealing yet another synergy in the underground world of experiments seeking weakly interacting particles (Horowitz, Coakley, \& McKinsey, 2003).

\section{EXCESS OF MATTER OVER ANTIMATTER IN THE UNIVERSE}

It is often not appreciated that the existence of normal matter in the universe is as stunning as that of dark matter. One should expect that there 
are equal amounts of matter and antimatter that would annihilate early on, leaving not much else than photons and neutrinos. In particle physics, we know that matter and antimatter act not quite the same, but what little differences we know are not enough to explain the cosmic excess of matter. One simple hypothesis proposes the existence of very heavy partners to the common neutrinos, explaining the smallness of neutrino masses. In this «see-saw model», the geometric mean of the new heavy neutrino masses and those of the common ones would be similar to ordinary quark and charged lepton masses. At the same time, the early-universe decays of these heavy neutrinos would naturally produce the cosmic matter-antimatter asymmetry, a process known as leptogenesis (Buchmüller, Di Bari, \& Plümacher, 2005).

One vestige of this model is the expectation that the common neutrinos are their own antiparticles. They are then said to be of Majorana type. This property enables a process called neutrino-less double beta decay (Dell'Oro, Marcocci, Viel, \& Vissani, 2016). Certain unstable nuclei decay by emitting two electrons («double beta») and two neutrinos at once, but might also do so without neutrinos if these are of Majorana type. One may say that one of the neutrinos is immediately reabsorbed as an antineutrino, leaving only the two electrons to emerge from the decaying nucleus. Searching for this rare phenomenon is another popular topic of underground physics, in Spain represented by the NEXT project in the Canfranc laboratory. ${ }^{3}$ Neutrino masses being as small as they are, this process is extremely hard to find.

\section{THE WEAK RULE IN HEAVEN}

While everyday phenomena on Earth are determined by electromagnetic and nuclear processes, the universe at large is dominated on the one hand side by gravity and on the other by weakly interacting particles. The identity of dark matter remains an unresolved mystery of the universe. Meanwhile neutrinos have matured from their status as ghost particles to distinctly visible messengers for some

\footnotetext{
3 http://next.ific.uv.es/next/
}

of the most intriguing astrophysical phenomena. Many of the experiments designed to unravel the remaining questions about neutrinos are also observatories for astrophysical neutrinos and notably for the next nearby supernova and its neutrino burst. Neutrino astronomy, similar to the recent beginnings of gravitational-wave astronomy, is a field in its infancy that will reveal a new panorama of the universe. (๑)

Physics, 2162659. doi: 10.1155/2016/2162659
DUNE Collaboration. (2015). Long-baseline neutrino facility (LBNF) and deep underground neutrino experiment (DUNE). Conceptual design report. Volume 2: The Physics Program for DUNE at LBNF. Retrieved from http://arXiv.org/abs/1512.06148

Gariazzo, S., Giunti, C., \& Laveder, M. (2016). Neutrino unbound. Retrieved from http://www.nu.to.infn.it/

Horowitz, C. J., Coakley, K. J., \& McKinsey, D. N. (2003). Supernova observation via neutrino-nucleus elastic scattering in the CLEAN detector. Physical Review D, 68, 023005. doi: 10.1103/ PhysRevD.68.023005

Hyper-Kamiokande Proto-Collaboration. (2015). Physics potential of a long-baseline neutrino oscillation experiment using a J-PARC neutrino beam and Hyper-Kamiokande. Progress of Theoretical and Experimental Physics, 053C02. doi: 10.1093/ptep/ptv061

IceCube Collaboration. (2013). First observation of PeV-energy neutrinos with IceCube. Physical Review Letters, 111, 021103. doi: 10.1103/ PhysRevLett.111.021103

Janka, H.-T. (2012). Explosion mechanisms of core-collapse supernovae. Annual Review of Nuclear and Particle Science, 62, 407-451. doi: 10.1146/annurev-nucl-102711-094901

JUNO Collaboration. (2016). Neutrino physics with JUNO. Journal of Physics G: Nuclear and Particle Physics, 43, 030401. doi: 10.1088/09543899/43/3/030401

Koshiba, M. (1992). Observational neutrino astrophysics. Physics Reports, 220, 229-381. doi: 10.1016/0370-1573(92)90083-C

Mirizzi, A., Tamborra, I., Janka, H.-T., Saviano, N., Scholberg, K., Bollig, R., ... Chakraborty, S. (2016). Supernova neutrinos: Production, oscillations and detection. La Rivista del Nuovo Cimento, 39, 1-112. doi: $10.1393 / \mathrm{ncr} / \mathrm{i} 2016-10120-8$

Scholberg, K. (2012). Supernova neutrino detection. Annual Review of Nuclear and Particle Science, 62, 81-103. doi: 10.1146/annurevnucl-102711-095006

Villaescusa-Navarro, F., Bull, P., \& Viel, M. (2015). Weighing neutrinos with cosmic neutral hydrogen. Astrophysical Journal, 814, 146-165. doi: $10.1088 / 0004-637 X / 814 / 2 / 146$

Georg G. Raffelt. Senior staff scientist at the Max Planck Institute for Physics in Munich (Germany). His research focuses on the areas of theoretical astroparticle physics and cosmology. One of his specialities is supernova neutrinos and neutrino oscillations in dense media. 